\title{
High-Power Semiconductor Laser with Diffraction-Limited Output Beam
}

\author{
Ching-Fuh Lin "a,", Chih-Hung Tsai ${ }^{c}$, Chia-Wei Tsai ${ }^{c}$, D. P. Tsai ${ }^{c}$ and Yi-Shin Su ${ }^{a}$ \\ "Graduate Institute of Electro-Optical Engineering \\ 'Department of Electrical Engineering and Graduate Institute of Electronics Engineering \\ "Graduate Institute of Physics \\ National Taiwan University \\ Taipei 106, Taiwan ROC \\ Tel: 886-2-23635251 ext. 339/Fax: 886-2-23638247 \\ Email: cflin(a)cc.ee.ntu.edu.tw
}

High-power semiconductor lasers could be used as pumping sources for solid-state lasers, fiber lasers, Raman lasers, and for material processing and medicine applications, etc. To increase the output power, broad-area laser diodes are usually used, but beam quality is easily degraded due to filamentation. Other methods like flared or tapered waveguides [1]-[3] have been used to improve beam quality. However, effects like spontaneous emission, [4] thermal lensing, and intrinsic property of semiconductor materials [5] etc. still degrade the beam quality. In this work, we discover a new broad-area waveguide structure that could result in high output power with diffraction-limited beam.

The structure has the broad-area waveguide similar to the conventional broad-area laser diodes, but the waveguide is aligned at $7^{\circ}$ from the normal of the cleaved facets. The waveguide is very braod. The width is $100 \mu \mathrm{m}$. Its length is $1.5 \mathrm{~mm}$. In the fabrication, reactive-ion-etching technique was used to create the ridge-type broad-area waveguide, which is critical and different from the usual broad-area laser diode. Etching was stopped at about $100 \mathrm{~nm}$ above the separate confinement hetero-structure layer of the epitaxial layers. Standard processing steps were applied for other fabrication steps. Due to the angled waveguide, the light path follows a zigzag line, which is similar to the path in angle-grating distributed feedback lasers. [6] However, the zigzag path in our devices is not due to reflection caused by the grating structures. Instead, it is due to the reflection of the boundaries of the ridge-type broad-area waveguide. We call the devices as angled broad-area semiconductor lasers because the waveguide is aligned not normal to the cleaved facets.

The angled broad-area semiconductor laser was measured with and without feedback from an external mirror. Because of the zigzag path, the light could propagate between the two cleaved facets and oscillate in the device. Therefore, the device could lase by itself without external feedback. At lasing, the output light mainly emits at the direction normal to cleaved facet. The FWHM divergence angle of the far-field intensity is about $5^{\circ}$. Another lobe of far-field intensity was also observed at an angle of $42^{\circ}$ from the facet normal. The near-field intensity was measured. The FWHM width is $77 \mu \mathrm{m}$, but the entire region of the near-field is more than $100 \mu \mathrm{m}$ probably because of the current spreading. When the angled broad-area semiconductor laser was setup in an external cavity with light emitted from one of the facets feedback by an external mirror, the output power was significantly increased. The output light could be more than $2 \mathrm{~W}$. The FWHM horizontal divergence angle is also reduced to as small as $0.7^{\circ}$. The second lobe of far-field intensity at the angle of $42^{\circ}$ from the facet normal almost disappeared when there was feedback. In the meantime, the near-field distribution is broadened to have a FWHM width of $160 \mu \mathrm{m}$, corresponding to diffraction limit.

The spectrum of the angled broad-area semiconductor laser was also measured. Before lasing, the spectrum is very broad because nonidentical multiple quantum wells [7] are used as the gain medium. The FWHM spectral width is $140 \mathrm{~nm}$, covering from $1500 \mathrm{~nm}$ to $1640 \mathrm{~nm}$. When the device lases, the spectral width is narrowed, but is still not a single frequency because there is no wavelength selection mechanism in the device except the gain dynamics. Without the external feedback, the spectrum covers from $1607 \mathrm{~nm}$ to $1620 \mathrm{~nm}$. With the external feedback, the spectrum covers from $1605 \mathrm{~nm}$ to $1626 \mathrm{~nm}$.

Compared to the usual broad-area or tapered waveguide lasers, the output beam is significantly improved. Because the phase front of the beam is not orthogonal to the waveguide direction, effects like thermal lensing, nonuniform current distribution across the waveguide, and so on do not always occur at the same lateral location of the beam, leading to the improved beam quality. The details will be discussed in the presentation.

\section{References:}

[1]S. O' Brien, R. Lang, R. Parke, J. Major, D. F. Welch, and D. Mehuys, IEEE Photonics Technol. Lett. 9, 440 (1997). [2]J. N. Walpole, Opt. Quantum Electron. 28, 623 (1996).

[3]Ching-Fuh Lin and Jie-Wei Lai, CLEO/Europe EQEC '96, Hamburg, Germany, Paper CThl29, 1996.

[4]S. Ramanujan and H. G. Winful, IEEE J. Quantum Electron., 32, 789 (1996).

[5]Jie-Wei Lai and Ching-Fuh Lin, IEEE J. Quantum Electron, 34, 1247 (1998).

[6]V. V. Wong, S. D. DeMars, A. Schoenfelder, and R. Lang, CLEO '98, Tech. Dig., 1998, pp.34-35.

[7]Ching-Fuh Lin, Yi-Shin Su, and Bing-Ruey Wu, CLEO '01, Tech. Dig., 2001, pp.237-238. 